\title{
Visual detection of rice rows based on Bayesian decision theory and robust regression least squares method
}

\author{
Jing He, Ying Zang , Xiwen Luo, Runmao Zhao, Jie He, Jinkang Jiao \\ (Key Laboratory of Key Technology on Agricultural Machine and Equipment, Ministry of Education, \\ South China Agricultural University, Guangzhou 510642, China)
}

\begin{abstract}
Paddy field management is complicated and labor intensive. Correct row detection is important to automatically track rice rows. In this study, a novel method was proposed for accurate rice row recognition in paddy field transplanted by machine before the disappearance of row information. Firstly, Bayesian decision theory based on the minimum error was used to classify the period of collected images into three periods (T1: 0-7 d; T2: 7-28 d; T3: 28-45 d), and resulting in the correct recognition rate was $97.03 \%$. Moreover, secondary clustering of feature points was proposed, which can solve some problems such as row breaking and tilting. Then, the robust regression least squares method (RRLSM) for linear fitting was proposed to fit rice rows to effectively eliminate interference by outliers. Finally, a credibility analysis of connected region markers was proposed to evaluate the accuracy of fitting lines. When the threshold of credibility was set at $40 \%$, the correct recognition rate of fitting lines was $96.32 \%$. The result showed that the method can effectively solve the problems caused by the presence of duckweed, high-density inter-row weeds, broken rows, tilting $\left( \pm 60^{\circ}\right)$, wind and overlap.
\end{abstract}

Keywords: rice rows detection, Bayesian decision theory, clustering, RRLSM, credibility analysis, automatic tracking DOI: $10.25165 /$ j.ijabe.20211401.5910

Citation: He J, Zang Y, Luo X W, Zhao R M, He J, Jiao J K. Visual detection of rice rows based on Bayesian decision theory and robust regression least squares method. Int J Agric \& Biol Eng, 2021; 14(1): 199-206.

\section{Introduction}

Rice is one of the most important cereal crops worldwide and is predominantly grown in the Asian monsoon region. Rice farming occupies 31 million $\mathrm{hm}^{2}$ in China, accounting for $20 \%$ of global production and representing a total rice yield of 208.56 million $\mathrm{t}$ in $2016^{[1]}$. With the rapid development of mechanized rice production, the total mechanization level for rice has reached $79.2 \%$, among which tillage, cultivation and harvest mechanization levels are $99.31 \%, 44.45 \%$, and $87.11 \%$, respectively ${ }^{[2]}$. In recent years, intelligent rice farming machinery has been developing, like the Global Navigation Satellite System (GNSS) based unmanned transplanter and unmanned combine harvester are becoming increasingly popular. However, paddy field management remains complicated and labor intensive and the mechanization level for this link is only $16.84 \%{ }^{[2]}$. Currently, weed control, fertilization, and pest control processes for paddy fields mainly adopt human-operated high-clearance machines, which have a low degree of automation. A human operator is typically required to concentrate on not driving over the rice rows; however, this is a quite difficult and tiring task (Figure 1a). Conversely, high-clearance paddy management machines navigated by machine

Received date: $2020-05-21 \quad$ Accepted date: $2020-10-18$

Biographies: Jing $\mathrm{He}, \mathrm{PhD}$, research interest: agricultural electrification and automation, Email: hejing_1127@163.com; Xiwen Luo, Professor, research interest: agricultural mechanization and automation, Email: xwluo@scau.edu.cn; Runmao Zhao, $\mathrm{PhD}$, research interest: agricultural mechanization and automation, Email: zhrm_2007@163.com; Jie He, PhD, research interest: agricultural mechanization and automation, Email: hooget@scau.edu.cn; Jinkang Jiao, $\mathrm{PhD}$, research interest: agricultural mechanization and automation, Email: jiaojk320@163.com.

*Corresponding author: Ying Zang, Professor, research interest: agricultural mechanization and automation. 483 Wushan Road, Tianhe District, Guangzhou, 510642, China. Tel/Fax: +86-20-38676975, Email: yingzang@scau.edu.cn. vision technology can automatically track rice rows, thereby significantly reducing the labor intensity and minimizing the damage to crop rows caused by tractor operation ${ }^{[3-7]}$. Correct row detection is crucial to automatic tracking, but machine vision-aided rice rows recognition technology faces the following challenges.

(1) The paddy field environment is complicated by different water depths in different fields, severe inverted image and mirror effects, difficulties to tell color feature between rice and weeds, duckweeds, cyanobacteria, and the presence of natural wind leading to the overlap of adjacent rice rows and unclear row information. At present, the researchers choosing different color spaces and color features for processing images can effectively reduce the influence of light intensity changes and weeds on image segmentation ${ }^{[8,9]}$. Moreover, Zhang ${ }^{[7]}$ proposed the smallest univalue segment assimilating nucleus (SUSAN) corner and improved sequential clustering algorithm, which can detect the rice row under the noise of cyanobacteria. Kaizu and Imou ${ }^{[10]}$ developed a dual-spectral camera system that could reduce water surface noise and clearly detect seedling rows. Furthermore, the appearance of weeds and crops can be differentiated by leaf shape and texture features as well ${ }^{[11-14]}$.

(2) Due to the unevenness of the bottom layer of paddy fields, paddy field machines and implements are forced to change position frequently ${ }^{[15-16]}$, which would lead to row breaking, tilting, and overlap. With the parallel and equidistant characteristics of crop rows, horizontal strip scanning can be used to search for feature points and fill the feature points into broken row space ${ }^{[17,18]}$. For some dry-land crops such as wheat, corn, and soybean, the horizontal strip scanning method can successfully detect crop rows in field with high-density weeds or even under varying circumstances like having different values of soil hardness, light intensity, and camera yaw; however, the method is hardly able to detect rows with uneven row spacing caused by the unevenness of the bottom layer of paddy fields during the mechanized 
transplanting process. Xiya et al ${ }^{[19]}$ proposed a position clustering algorithm including the distance threshold and angle threshold, which could effectively solve the problem of broken rows without overlap.
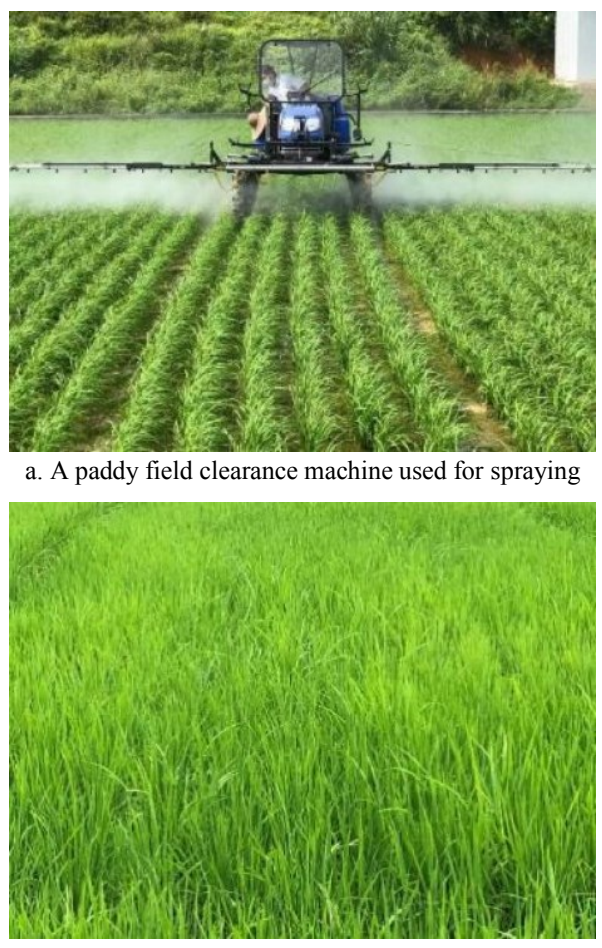

b. A paddy field in the closing stage

Figure 1 Paddy filed pictures from the Experimental Research

Farm, South China Agricultural University, Ningxi County, Guangdong Province, China $\left(23^{\circ} 16^{\prime} \mathrm{N} 113^{\circ} 22^{\prime} \mathrm{E}\right.$ and $11 \mathrm{~m}$ from the mean sea level)
(3) When standing at the ridge of a field, there is no water or ground in the paddy field would be seen from to a distance of $1.5 \mathrm{~m}$, the paddy field is in the closing stage (Figure 1b) ${ }^{[20]}$. The duration of the rice growth period suitable for machine vision detection is before the closing stage, which is approximately 45 days after transplanting and nearly covers the seedling stage and tillering stage $^{[21]}$. Throughout the above two periods, the plant density, height, and inter-row spacing of the rice vary significantly. Jiang et al. ${ }^{[22]}$ proposed an algorithm based on particle swarm optimization (PSO) clustering to detect crop rows of the over-wintering stage and re-growth stage of wheat rows, which achieved a recognition rate of $95 \%$. García-Santillán et al. ${ }^{[6]}$ detected corn rows under a row spacing range of 0.75-0.95 m. Choi et al. ${ }^{[23]}$ proposed an algorithm to identify the central region of the rice plant using the morphological characteristic, and Liao J et al. ${ }^{[24]}$ proposed a detection method of centerlines of rice seedling row based on sub-regional feature points clustering. However, the rice row detection of the above two mentioned methods did not take the overlap into consideration, and there were few noises in the image such as weeds and projection. Chen et al. ${ }^{[25]}$ proposed a method based on the automatic accumulation threshold of Hough Transformation, which can improve the adaptability of the crop row recognition algorithm for different kinds and growth periods of vegetables, but the time consumption for optimal accumulator threshold algorithm was about $1.5 \mathrm{~s}$.

As noted in previous studies, the problems of plant tilting, row breaking, image distortion, and significant changes in the plant morphology, which can all be aggravated by natural wind, have not yet been well solved in the complex paddy field environment. Therefore, this study proposed a method for identifying rice rows addressing above mentioned problems and verified the method using images taken from paddy fields (Figure 2a).

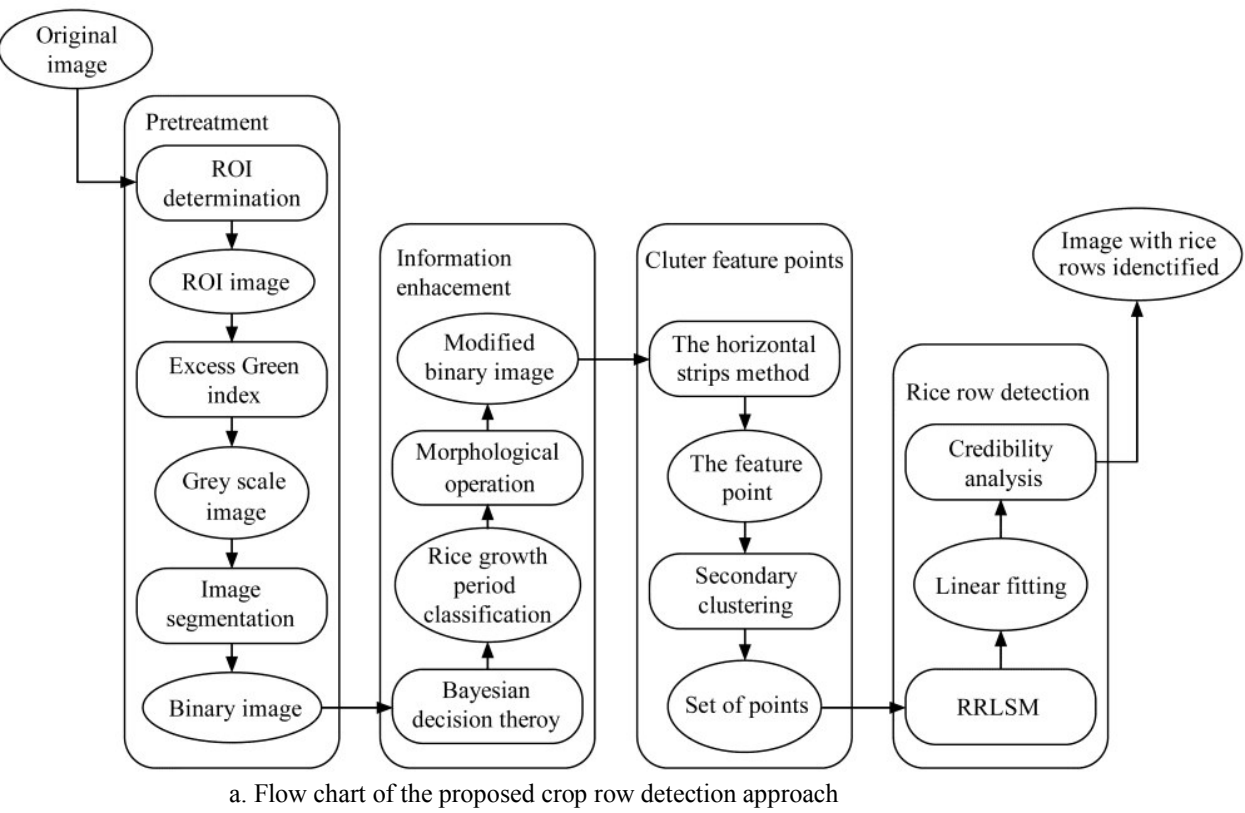

Figure 2 Flow chart and information collecting device

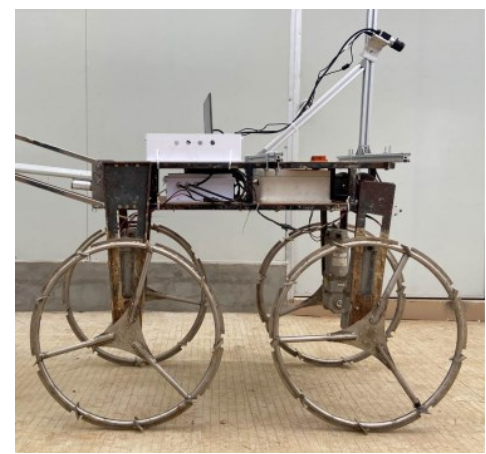

b. Remote-control vehicle carrying the camera and computer, with a battery for power supply

\section{Rice row recognition method}

\subsection{Image acquisition and pretreatment}

To illustrate and verify the row recognition method proposed in this study, 1200 images were collected from paddy fields transplanted by machine before the closing stage under different environments in Guangdong, Guizhou, and Jiangsu Provinces in
April and August 2019 (Figure 3). The inter-row spacing was about $30 \mathrm{~cm}$, and the images were collected on sunny or cloudy days at different times. The seed of rice cultivars in Guangdong, Guizhou and Jiangsu Provinces were 'Meixiangzhan-2', 'Yanjing-5' and 'C-liangyouhuazhan', respectively. In order to facilitate subsequent perception of crop growth, the best available settings (including resolution) were used. A BASLER 
acA4600-7gc color camera with a resolution of $4608 \times 3288$ pixels was used. It was showed in Figure $2 b$, the camera placed on a machine and the angle between the optical axis of the camera and the horizontal line was $30^{\circ}$. According to the clear differences of rice row width among different growth stages, rice images were divided into three categories characterized by the time after transplanting. $\quad T 1$ corresponds to days 0-7 after transplanting (Figure 3a), $T 2$ corresponds to days 7-28 (Figures 3b, 3c, 3d), and $T 3$ covers the remaining days before the closing stage (Figure $3 \mathrm{e}$, $3 \mathrm{f}$ ). The number of images collected for each period was $T 1=400$, $T 2=550$, and $T 3=250$. All methods were implemented in MATLAB R2018a ((MathWorks). The images were saved in red, green, and blue (RGB) color space in JPEG format. In order to reduce the processing time, the fixed red rectangular box was used to represent the Region of Interest (ROI, 550:4058, 150:3138), thus the image size was reduced to $1754 \times 1494$ pixels. In order to separate the rice rows from the paddy background, the ROI was extracted using the Excess Green (ExG) index, the index was computed as $\mathrm{ExG}=2 \mathrm{G}-\mathrm{R}-\mathrm{B}$. Then, the Otsu algorithm ${ }^{[26]}$ was used to binarise the images, which selected an optimal threshold value to split grey-level histogram of an image into two parts based on the principle of maximum between-cluster variance and minimum within-cluster variance. Next, Median filtering $(12 \times 12$ pixels) was applied to remove small patches and erroneous pixels from the binary image.

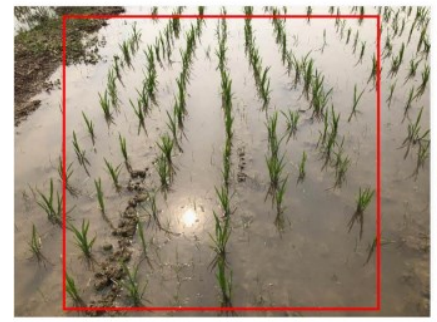

a.
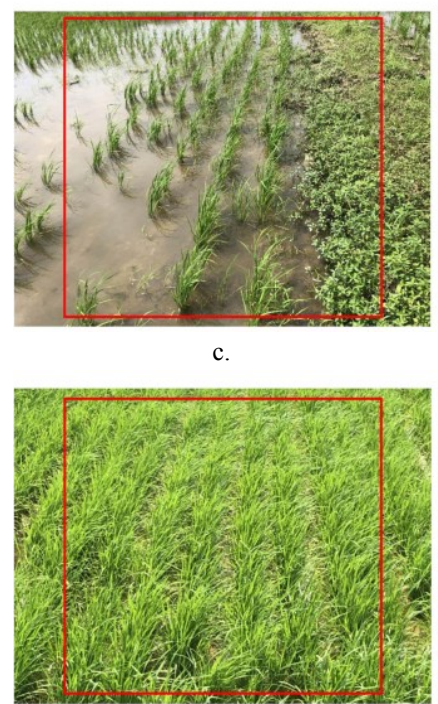

e.

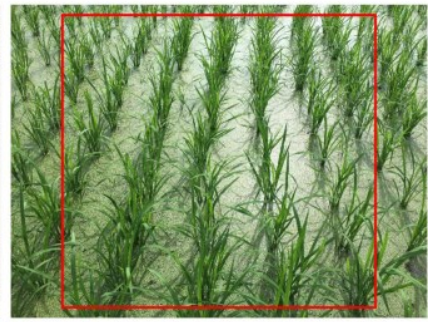

b.
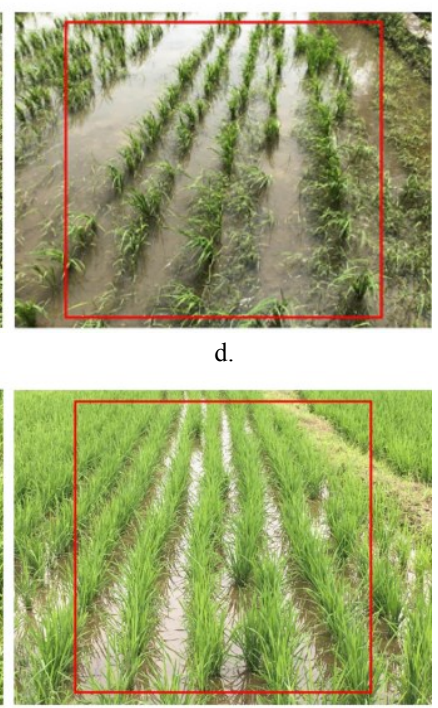

f.
Figure 3 Typical images of rice rows, where the red rectangle is the region of interest (a) $T 1$, showing row breaking; (b)-(d) $T 2$, showing the presence of duckweeds in (b), showing the presence of ridge weeds in (c), and showing the presence of inter-row weeds in

(d); (e)-(f) T3, showing the impact of wind in (e)

\subsection{Rice growth period classification and rice row} information enhancement

Correct classification of images according to rice growing periods can improve the recognition rate. In this study, based on the principle of minimizing classification error, the Bayesian formula (Equation (1)) was used to classify the rice images.

$$
P\left(\omega_{i} \mid X\right)=\frac{P\left(X \mid \omega_{i}\right) P\left(\omega_{i}\right)}{\sum_{j=1}^{M} P\left(X \mid \omega_{i}\right) P\left(\omega_{i}\right)} \cdots
$$

where, $M$ is the number of sample categories, $M=3 ; P\left(\omega_{i}\right)$ is the prior probability of class $\omega_{i}$, i.e., the ratio of the number of training samples belonging to class $\omega_{i}$ to the total number of training samples; $P\left(X \mid \omega_{i}\right)$ is the conditional probability density and $P\left(\omega_{i} \mid X\right)$ is the posterior probability expressed as the probability belonging to the class $\omega_{i}$.

Assuming the samples obey a normal distribution ${ }^{[28]}$, the discriminant model is described as follows:

$\ln g_{i}(X)=$

$-\frac{1}{2}\left(x-\mu_{i}\right) \sum_{i}^{-1}\left(x-\mu_{i}\right)^{T}+\ln P\left(\omega_{i}\right)-\frac{1}{2} \ln \left|\sum_{i}\right| \ldots(i=1, \ldots, M)$

where, $g_{i}(X)=P\left(X \mid \omega_{i}\right) P\left(\omega_{i}\right), \mu_{i}$ and $\Sigma_{i}$ are the mean value and covariance matrix of the class $\omega_{i}$, respectively, and $\Sigma_{i}^{-1}$ and $\left|\Sigma_{i}\right|$ are the inverse and determinant of $\Sigma_{i}$, respectively.

Based on the principle of minimizing classification errors, the posterior probability $P\left(\omega_{i} \mid X\right)$ should be the largest. If $g_{i}(X)=$ $\max _{i=1}^{M} g_{i}(X)$, then sample picture is classified in class $\omega_{i}$.

The classification data of the minimum error Bayesian decision was the mean width of rice rows in the image. Based on the perspective principle, rice row information was clearer at the bottom of the image. The origin of coordinates was the top left corner of the image. Hence, the position $1 / 2 h, 2 / 3 h$, and $5 / 6 h$ were selected as scanning lines to extract rice row width data, where $h$ was the heigh of images, in this study $h=1494$ pixels.

A total of 500 images were selected as the training set, which included 100 images, 210 images, and 190 images corresponding to $T 1, T 2$, and $T 3$, respectively. Rice row width data of the training set was extracted, then the mean $\mu_{i}$, covariance inverse matrix, $\Sigma_{i}^{-1}$, covariance determinant, $\left|\Sigma_{i}\right|$, and prior probability, $P\left(\omega_{i}\right)$, of above three sample class were calculated (Table 1). Next, a total of 539 images were selected as the testing set, and the testing set was classified based on the discriminant model (Equation (2)).

According to the obvious characteristics of rice row information in different periods, different morphological operations were used to enhance the rice row information. The information of each rice row in $T 1$ is independent, exhibiting clear interplant distances and rice seedlings expand outward from their flag leaves. The dilation process can fill the gaps in the plants ${ }^{[6]}$. Therefore, to ensure that the feature points were located in the center of the rice seedling, $20 \times 20$ structural elements were selected for the dilation operation for $T 1$ images. In $T 2$, the intersection of the leaf tips appears between rows. Although the width of rice rows become wider for tillering, the inter-row spacing remains clear. The erosion can eliminate small connections between objects $^{[29]}$. Therefore, $T 2$ images were processed by erosion, opening $^{[30]}$, and dilation operations, for which $50 \times 1,1 \times 20$, and $20 \times 20$ structural elements were selected, respectively. In $T 3$, the rice is in a later tillering stage. The width of the rice row continues growing, the intersection of leaf tips becomes more severe, and inter-row spacings are decreasing. Hence, these images were processed by erosion, opening, and dilation operations, for which $60 \times 1,1 \times 30$, and $5 \times 5$ structural elements were selected, respectively. 
Table 1 Parameters for the discriminant function

\begin{tabular}{|c|c|c|c|}
\hline Parameter & $T 1$ & $T 2$ & $T 3$ \\
\hline$\mu_{i}$ & {$[37.33,29.81,22.09]$} & {$[91.41,75.94,65.89]$} & {$[208.05,179.48,153.98]$} \\
\hline$\Sigma_{i}^{-1}$ & {$\left[\begin{array}{ccc}0.0451 & -0.0615 & -0.0069 \\
-0.0615 & 0.1046 & -0.0162 \\
-0.0069 & -0.0162 & 0.0458\end{array}\right]$} & {$\left[\begin{array}{ccc}0.0080 & -0.0053 & -0.0048 \\
-0.0053 & 0.0238 & -0.0195 \\
-0.0048 & -0.0195 & 0.0302\end{array}\right]$} & {$\left[\begin{array}{ccc}0.0009 & -0.0011 & -0.0001 \\
-0.0011 & 0.0025 & -0.0014 \\
-0.0001 & -0.0014 & 0.0026\end{array}\right]$} \\
\hline$\left|\Sigma_{i}\right|$ & $8.4324 \mathrm{e}+04$ & $3.1316 \mathrm{e}+06$ & $1.1826 \mathrm{e}+09$ \\
\hline$P\left(\omega_{i}\right)$ & 0.20 & 0.42 & 0.38 \\
\hline
\end{tabular}

\subsection{Rice row feature point extraction}

To reduce the computational consumption, the images after morphological operations (Figure 6) were then divided into 50 horizontal strips. The image histograms of each horizontal bar were obtained respectively, and the rice row boundary was found by Equation (3) and Equation (4) $)^{[31]}$. Then, Equations (5) and (6) were used to obtain the center location, Point, and average spacing $D$ of rice rows in the horizontal strip. In order to eliminate false centers point, a threshold $T$ was set. When $d i$, the distance between the point and the previous center point was greater than the threshold $T$, then the point was the real center location, which was stored in the array Media as the feature point (as shown in Figure 4, red dot). When $d i$ was less than the threshold $T$, the point was removed and not stored as the feature point. For example, in Figure 4, $d 7<T$, then the black symbol should be removed. Therefore, the number of columns in array Media was the actual number of rice rows in the image, $w \_$data, and the rice rows average spacing of the whole image calculated by Equation (7) was ready for clustering threshold setting. According to the statistics, the inclination angle $\beta$ of rice row in the image caused by camera lateral offset was not more than $60^{\circ}$. The size of the image was $1754 \times 1494$, and the maximum number of rice rows in the most collected image was 8 columns by observation, so the value of $T$ was about equal to $0.76 * D$ by Equation (8).

$$
\begin{aligned}
& H \text { Hum_ave }(t, j)= \begin{cases}1 & H \operatorname{Humpiex}(t, j)>\operatorname{aveHSum}(t) \\
0 & H \operatorname{Humpiex}(t, j) \leq \operatorname{aveHSum}(t)\end{cases} \\
& \text { if HSum_ave }(t, j)=0 \text {, and HSum_ave }(t, j+1)=1 \text {, } \\
& \text { so Point_left }=j \\
& \text { if HSum_ave }(t, j)=1 \text {, and HSum_ave }(t, j+1)=0 \text {, } \\
& \text { so Point_right }=j \\
& \text { if HSum_ave }(t, j)=1 \text {, and } j=1 \text {, so Point_left }=j \\
& \text { if num_left }>\text { num_right, and } j=w \text {, so Point_right }=j \\
& \text { Point }=\frac{(\text { Point_right }+ \text { Point_left })}{2} \\
& D=\frac{\text { Point }(\text { end })-\text { Point }(1)}{\text { num_right }} \\
& \text { all_average }=\frac{(w-\min (\text { Media }))}{w_{-} \text {Data }} \\
& T=\left(D-\tan \beta \cdot b a r_{-} \text {width }\right) \approx\left(1-\tan \beta \cdot \frac{h}{D \cdot \text { num }}\right) \cdot D
\end{aligned}
$$

where, HSumpiex $(t, j)$ is the sum of the pixel value of column $j$ in strip $t \quad(0<t \leq 50)$; aveHSum $(t)$ is the average pixel value; HSum_ave $(t, j)$ is the filtered value of column $j$ in strip $t$; Point_left, Point_right, num_left, and num_right are respectively the left boundary points, right boundary points, number of left boundary points, and number of right points in the horizontal strip $t$; bar_width is the width of each horizontal bar; num $=50$, is the number of horizontal bars; and $w$ is the width of the picture, in this paper $w=1754$ pixels.

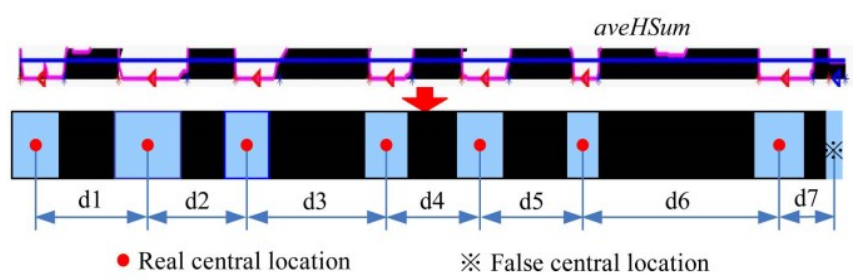

Figure 4 Acquisition of feature points in the target area

2.4 Secondary clustering of feature points based on shortest distance method

The shortest distance algorithm is a common method for clustering feature points in crop row recognition ${ }^{[7,19,28]}$. At first, find the seed points row i0 and set seed points as cluster center. As the rice row was broken, it was easy to happen that one or more rice rows were omitted when one fixed row was selected as seed point row. The actual number of rice rows in one image $w$ data was a known parameter, so the Media was scanned to find the row i0 in which there was no zero elements and was closest to $h / 2$. Next, the distance between the feature point and cluster center was calculated by Euclidean distance. At last, each feature point was clustered in turn based on the principle of shortest distance, and the cluster center was updated.

The broken rows, overlap, and tilting can all lead to the first clustering coming out unsatisfactory results. Moreover, not all rows ended at the bottom edge in the images, as shown in Figure 5, the left-most rice row ended at point 1 but the Euclidean distance between point 2 (in the adjacent rice row) and point 1 was less than that between point 2 and point 3 (in the same row). In order to solve the above problems, the second clustering method which was based on the result of the first clustering was proposed.

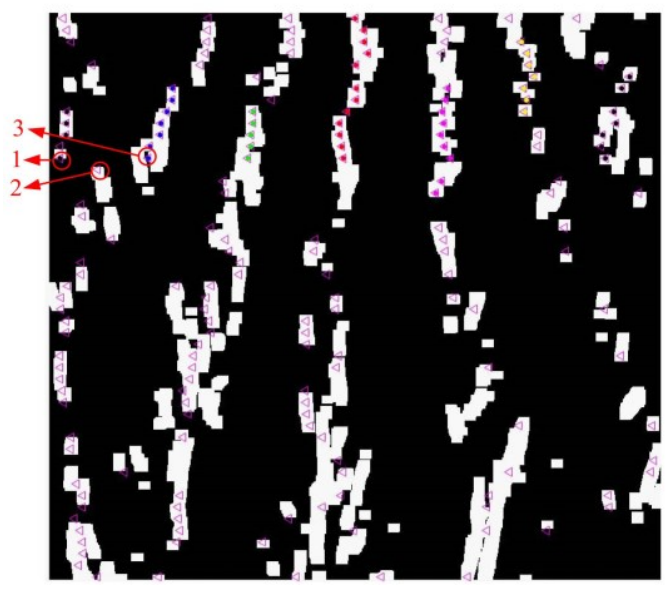

Figure 5 An example of rice rows ending in the middle of the image

The process of the second clustering algorithm was as follows:

(1) Find possible clustering number. Scan each horizontal 
image, and find the feature points without clustering. Based on the first clustering results, the possible clustering number was judged and stored in the array zero_point.

(2) Remove the finished clustering number. The finished clustering number was saved in the array line_number, and delete the intersection of zero point and line_number from the array zero point.

(3) Find the shortest distance clusters. To solve the problem of row breaking, only the $X$-axis direction distances were considered from Media $(i, j)$ to the cluster center cluster center(i-1,zero point), find the nearest column $j 1$ and calculate its corresponding distance distancce 2 .

(4) Threshold judgement. Set $T$ _distance $2=K X 2 *$ all_average $(K X 2=[0.5,0.6,0.7])$. If distance $2<T \_$distance 2 , then Media $(i, j)$ was clustered in class $j 1$, update the cluster center point.

(5) Determine whether this clustering number had been finished in the middle of the image, i.e. determine whether this rice row ended. A row ending was characterized by an $X$-axis located close to the left or right boundary points ( 0 or w). Ideally, the displacement of adjacent horizontal bars (upper and lower) $D_{T_{-} \text {line }}=b a r_{-}$width $* \tan \beta$. In this study, the maximum bending angle, $\beta$, was considered $60^{\circ}$, which corresponded to about 51 pixels. Therefore, set $T$ line $=50$; if Data $(i, j 1)<T$ line or $w$-Data $(i, j 1)<T$ line, then line_number $=[$ line_number, $j 1]$.

(6) This process was repeated until the second-to-last point ended the clustering.

\subsection{Robust regression least squares method (RRLSM) for} linear fitting

The least squares method (LSM) and Hough transform (HT) are commonly used linear fitting methods in crop row recognition. The HT method is time-consuming ${ }^{[22]}$, whereas the LSM assigns the same weight to all sample data; thus, even outliers are processed as normal values and are very sensitive to parameter estimates $^{[32]}$. After the above process, there were inevitably a few outliers. Hence, the robust regression least squares method (RRLSM) was proposed to perform line fitting in this part to eliminate outliers ${ }^{[32-34]}$. Different weights, $\zeta_{i}$, to different feature points (Equation (9), Equation (10)) were assigned. The greatest advantage was that the estimators generated during the estimation process were insensitive to model errors, which effectively eliminated interference by outliers.

$$
\begin{gathered}
\sum_{i=1}^{n} \zeta_{i}\left(y_{i}-\sum_{j=1}^{p} x_{i j} \beta_{j}\right)^{2}=\min \\
\zeta_{i}= \begin{cases}1, & \left|u_{i}\right| \leq c_{h} \\
c_{h} /\left|u_{i}\right|, & \left|u_{i}\right|>c_{h}\end{cases}
\end{gathered}
$$

\subsection{Credibility analysis of connected region markers}

The accuracy of fitting rice rows was uneven. In addition, if there was no broken row, each rice row should correspond to one connected pixel area and should have feature points in each horizontal strip. According to these characteristics, credibility analysis based on the integrity of feature points in the connected regions was proposed to assist the selection of the optimal precision fitting lines from the lines with different precision. Firstly, the connected regions of images were labeled after morphological processing. Then, the sum of feature points sum point and the largest proportion of label points number L_point were counted separately in each cluster group. At last, the parameter credit_rate, representing the credibility of each fitting line, was calculated using Equation (11).

$$
\left\{\begin{array}{l}
L_{-} \text {rate }=\frac{L_{-} \text {point }}{\text { num }} \\
\text { point_rate }=\frac{\text { sum_point }}{\text { num }} \\
\text { credit_rate }=\frac{\left(L_{-} \text {rate }+ \text { point_rate }\right)}{2} \times 100 \%
\end{array}\right.
$$

\section{Results and discussion}

(1) The results of information enhancement based on rice growth period classification

The Bayesian decision theory based on the minimum error was proposed to divide the binary images into three growth periods, and the number of false judgments was 16 and the correct recognition rate was $97.03 \%$. Furthermore, due to the outward expansion of the flag leaves of rice seedlings, different linear morphological operations were performed on images for different periods. As such, rice row information was highlighted from inter-row interference (Figure 6, corresponding to each one in Figure 3). During the whole period of rice mechanical management, from the time of $T 1$, the weak information of early rice seedling, to the time of $T 3$, the adjacent rice rows crossed with each other and the space between the two rows disappeared. This method can eliminate the impact of duckweeds (Figure 6b), inter-row weeds (Figure 6d), and wind (Figure 6e), and significantly enhance and highlight rice row information.
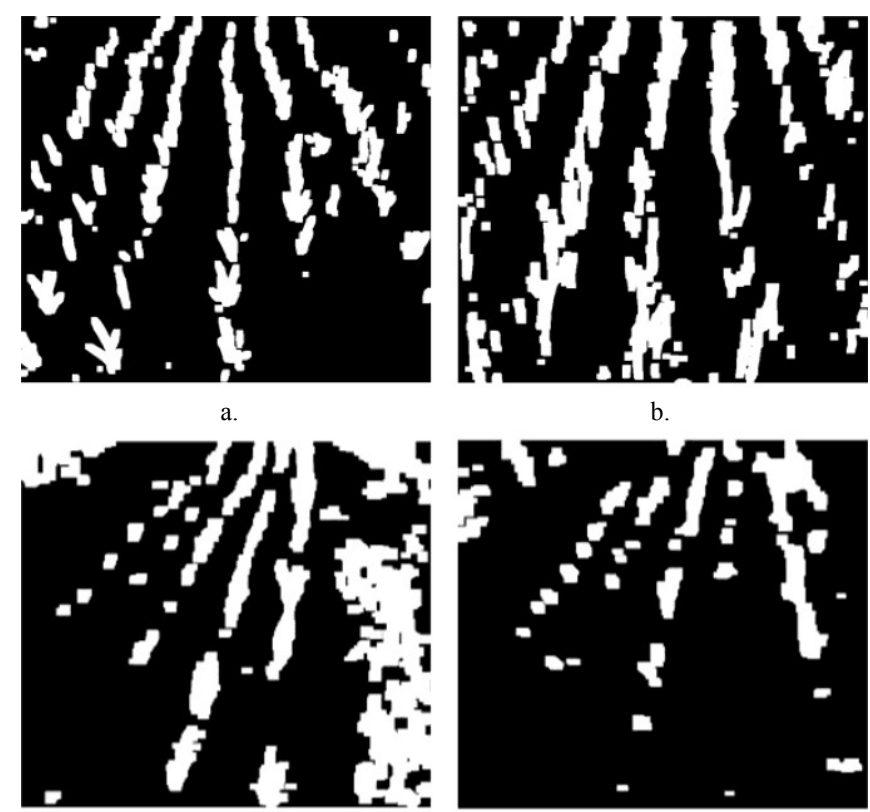

c.

d.
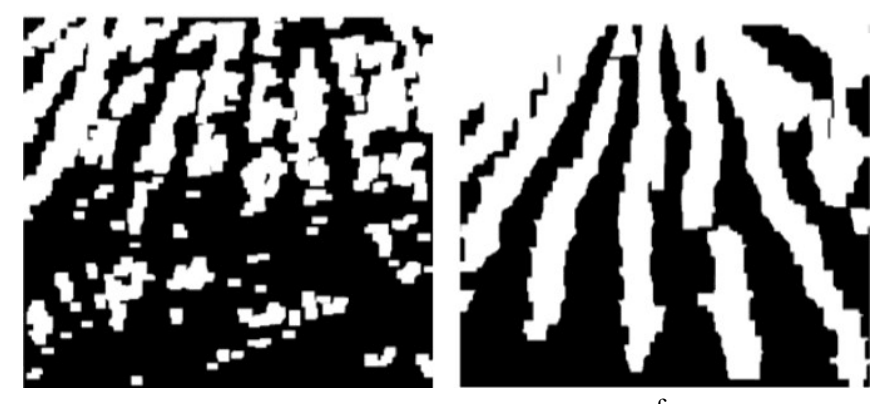

Figure 6 Information enhancement of typical images (a) $T 1$, showing row breaking, (b)-(d) $T 2$, showing the presence of duckweeds in (b), showing the presence of ridge weeds in (c), and showing the presence of inter-row weeds in (d), and (e)-(f) T3, showing the impact of wind in (e) 
(2) Secondary clustering of feature points based on the shortest distance method

After extracting the feature points by the vertical projection method, seed points were automatically selected, and the image was divided into upper and lower parts, whose feature points were clustered by the shortest distance method. Rice row breaking and ending in the middle of images can all easily lead to leakage points after the first clustering. As shown in Figure 7a, few of the feature points were correctly clustered, while the secondary clustering method proposed in this paper can find most of those unclustered points and cluster them correctly (Figure 7b).

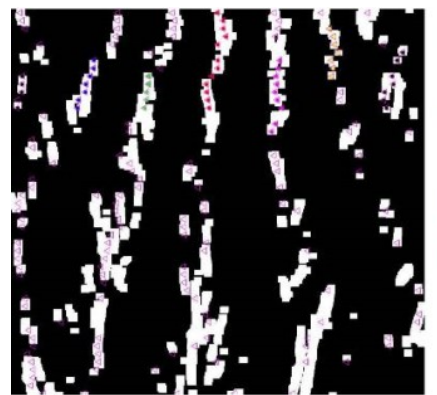

a. The result of first clustering

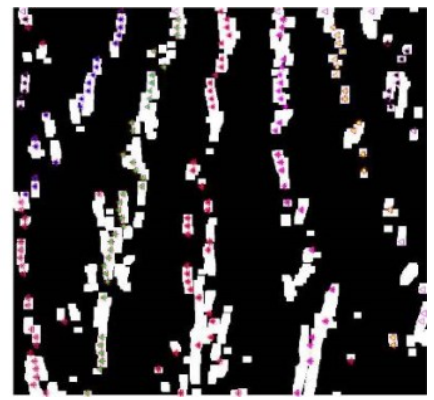

b. The result of second clustering
Figure 7 Comparison of the results between the first and second clustering

(3) Robust regression least squares method for linear fitting

As shown in Figure 8, an image with 7 rice rows (size $1754 \times 1494$ pixels) was select, the upper and lower center points in each rice row were found to connect them into a straight line which was taken as the reference line of the actual rice row. Lines fitted by RRLSM, LSM and HT were drawn in the images. Compared with 7 rows fitted manually, the maximum, minimum, and average

absolute errors using RRLSM were $2.820^{\circ}, 0.050^{\circ}$, and $1.561^{\circ}$, respectively, while that using LSM method were $6.120^{\circ}, 0.450^{\circ}$, and $1.971^{\circ}$, respectively, and that using HT method were $16.890^{\circ}$, $1.210^{\circ}$, and $5.162^{\circ}$, respectively. To process an image with $1754 \times 1494$ pixels, the computational time for RRLSM, LSM and HT were $25.7 \mathrm{~ms}, 10.3 \mathrm{~ms}$ and $255 \mathrm{~ms}$, respectively. The result showed that RRLSM had minimum errors and could accurately express rice row information. Moreover, RRLSM had strong real-time performance in time-consuming.

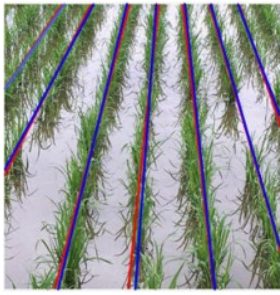

a. RRLSM

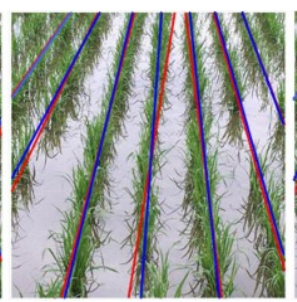

b. LSM

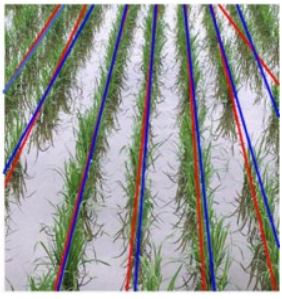

c. HT

Figure 8 Comparison of crop rows detected by various fitting methods (red lines) and real rows fitted manually (blue lines)

The final rice row detection results for the images in Figure 3 were shown in Figure 9. Multiple rice row lines in each image were fitted, and the fitting results were basically accurate. However, as shown in Figure 9c, the right side with high-density weed was fitted as straight lines; In Figure 9f, the different inclinations of rice row contributed to different precision of fitting straight lines. Hence, this study proposed the credibility analysis which was based on the connected regions, and the results showed that the credibility of the right ridge with high-density weed in Figure 9c was 35.71\%, and the credibility of the offset fitting line (left 1) in Figure 9f was $35.42 \%$.

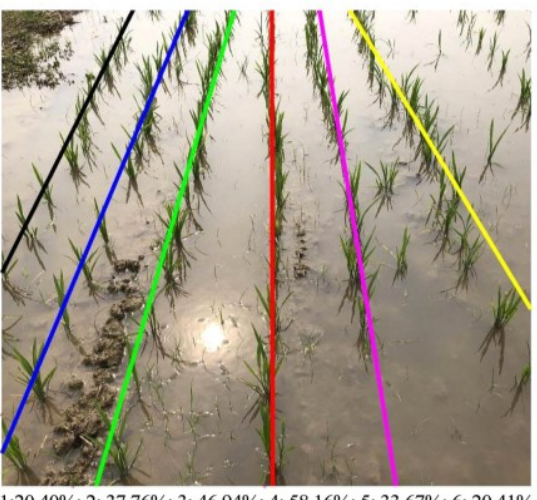

$1: 20.40 \% ; 2: 37.76 \% ; 3 ; 46.94 \% ; 4: 58.16 \% ; 5: 33.67 \% ; 6: 20.41 \%$

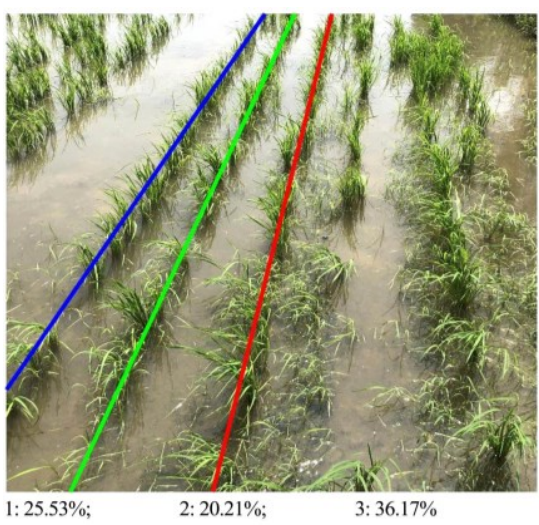

d.

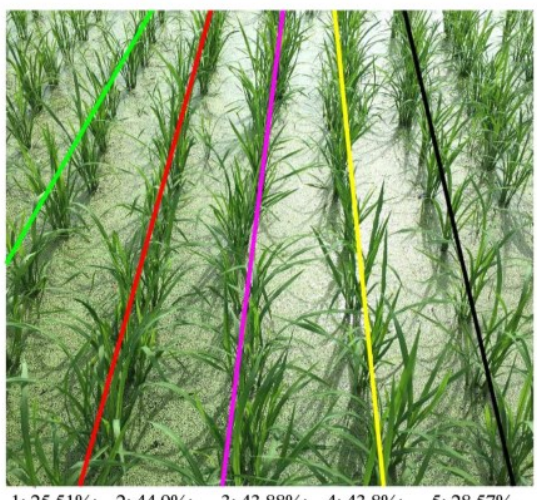

b.

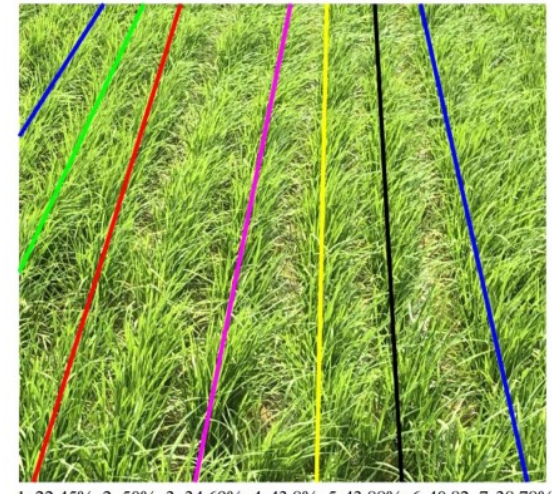

1: $22.45 \% ; 2: 50 \% ; 3: 34.69 \% ; 4: 43.8 \% ; 5: 43.88 \% ; 6: 40.82 ; 7: 38.78 \%$

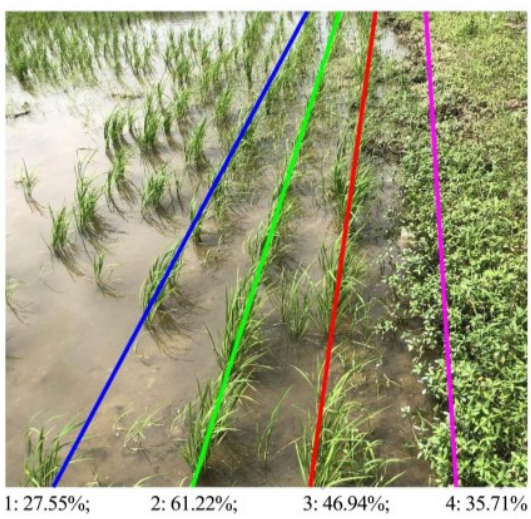

c.

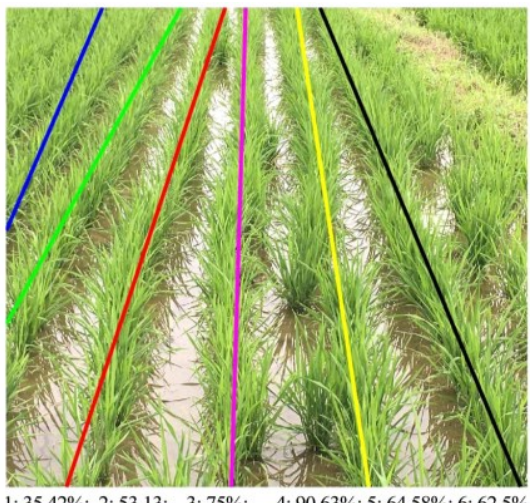

f.

Figure 9 Final rice row detection results for the images in Figure 3 (a) $T 1$, showing row breaking, (b)-(d) $T 2$, showing the presence of duckweeds in (b), showing the presence of ridge weeds in (c), and showing the presence of inter-row weeds in (d), and (e)-(f) $T 3$, showing the impact of wind in (e) 
(4) Results of crop row recognition before the closing stage

Under different natural environments, 1200 paddy field images were collected from fields planted by machine, and the number of images collected for each time period was $T 1=400, T 2=550$, and $T 3=250$. When taking the credibility no less than $40 \%$ as a judgment basis, the average correct recognition rate was $96.32 \%$, and the recognition rates of $T 1, T 2$ and $T 3$ were $98.75 \%, 99.00 \%$ and $91.20 \%$, respectively. The main reason for the obvious decrease of recognition rates of $T 3$ was that the adjacent rice rows crossed seriously in the later period, very close to the closing stage. As shown in Figure 10, under different circumstances such as uneven planting, bent rows, the presence of inter-row weeds, the overlap caused by wind, and the inclination angle of rice less than $60^{\circ}$ caused by camera lateral offset, the rice row all can be detected correctly. Hence, the rice row recognition method proposed in this paper can meet the requirements of rice row recognition and location for fields planted by machine in the complex paddy field environment.

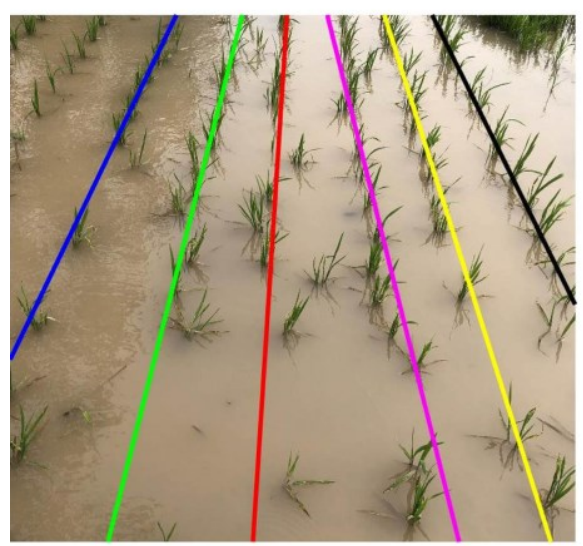

1: $22.45 \% ; 2: 32.65 \% ; 3: 27.55 \% ; 4: 29.59 \% ; 5: 25.51 \% ; 6: 24.49 \%$

a.

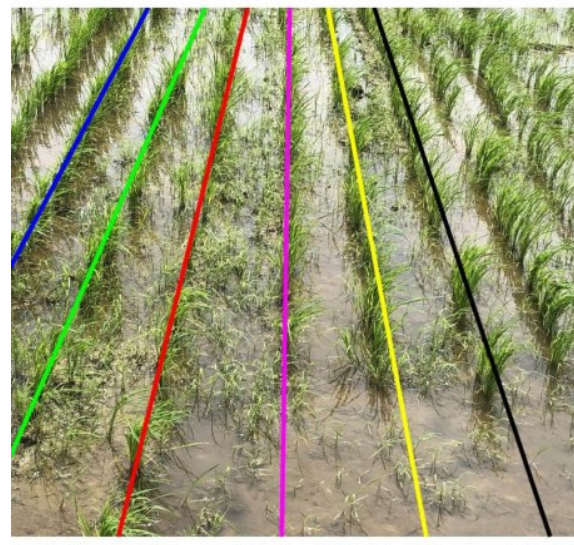

$1: 43.88 \% ; 2: 39.8 \% ; \quad 3: 48.98 \% ; 4: 53.06 \% ; 5: 33.67 \% ; 6: 44.9 \%$

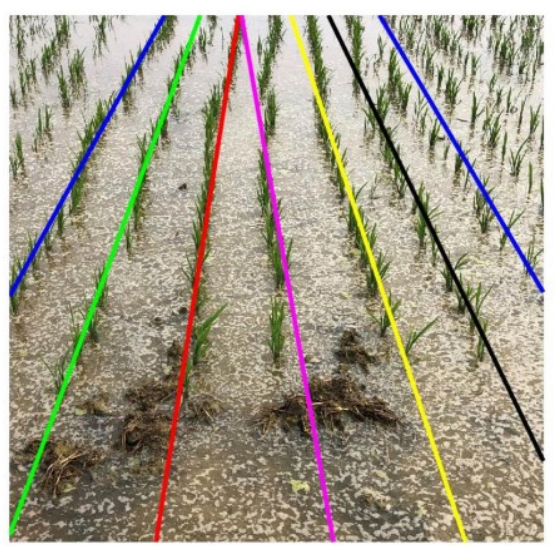

1: $77.78 \% ; 2: 72.22 \% ; 3: 98.61 \% ; 4: 81.94 \% ; 5: 80.56 \% ; 6: 63.89 \% ; 7: 23.61 \%$

b.

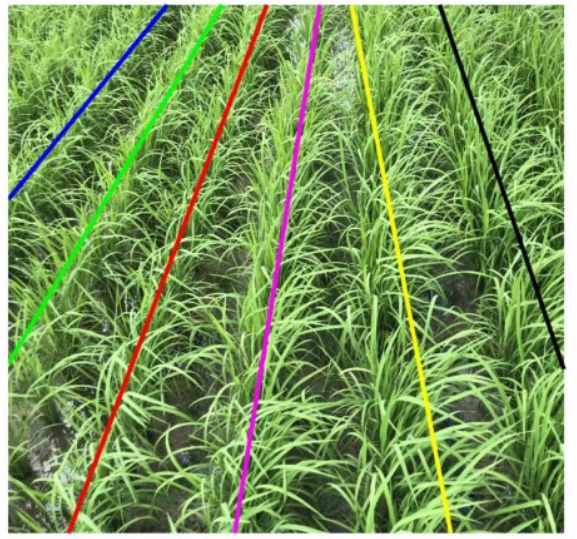

$1: 20.83 \% ; 2: 26.04 \% ; 3: 20.83 \% ; 4: 40.63 \% ; 5: 33.33 \% ; 6: 33.33 \%$
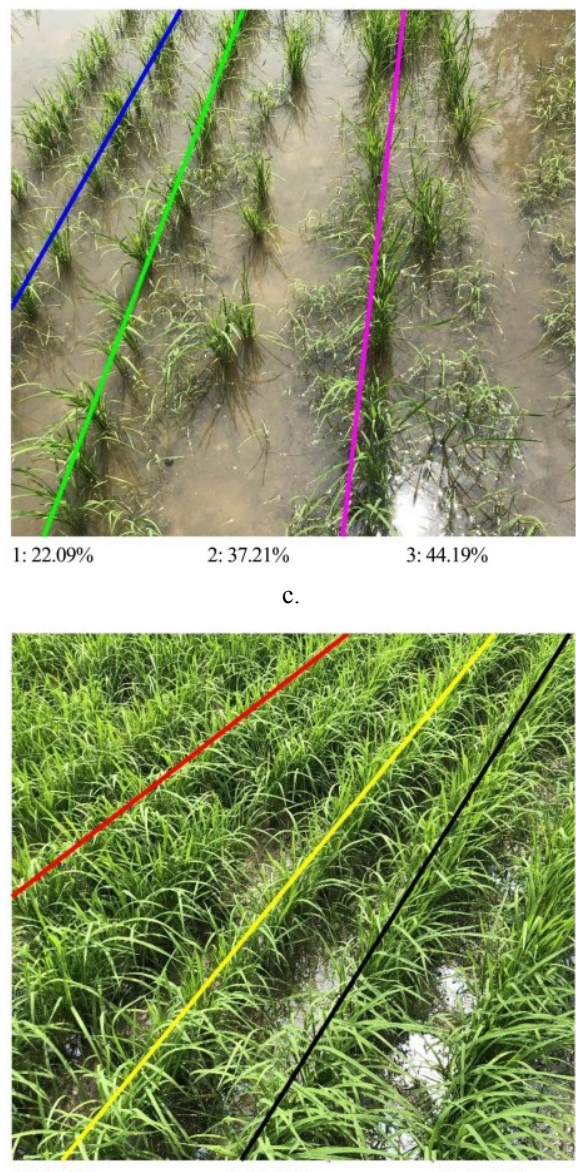

1: $24.49 \%$;

f.

Figure 10 Overall rice row detection results for complex conditions (a) Uneven planting, (b) bent rows, (c) and (d) the presence of inter-row weeds, (e) the effect of wind, and (f) camera lateral offset

\section{Conclusions}

In this study, a novel method for accurate crop-row detection in paddy fields before the closing stage was proposed. The proposed method consists of four main processes: rice growth period classification, feature point extraction, crop row detection and credibility analysis. Firstly, the Bayesian decision theory based on the minimum error ratio was used to divide the rice images into three time periods, and different linear morphological operations were performed on images to enhance the rice row information. Then, the vertical projection method was combined with horizontal strip division to identify the feature points of the crops. Next, secondary clustering of feature points based on the shortest distance method was proposed, which effectively improved the number of correct cluster points, and the rice rows with tilting angle (within $\pm 60^{\circ}$ ) could also be clustered. The RRLSM effectively eliminated the impact of outliers and accurately fitted the rice row. Finally, credibility analysis of connected region markers was proposed to assist the selection of the optimal precision fitting lines from the lines with different precision. The performance of the proposed method was tested using a set of images, and this experiment results showed that the correct recognition rate of images was $96.32 \%$. However, the average computational time from reading one image $(1754 \times 1494$ pixels $)$ to credibility analysis was about $0.8 \mathrm{~s}$. The optimization of processing time will be paid more attention in the future.

\section{Acknowledgements}

This work was financially supported by the Key-Area Research and Development Program of Guangdong Province (Grant No. 2019B020221002) and the National Key Research and Development Program of China (Grant No. 2017YFD0701105). The authors also acknowledge the anonymous reviewers for their critical comments and suggestions for improving the manuscript.

\section{[References]}

[1] Yi Z Y. China Agricultural Mechanization Yearbook 2017. China Agricultural Science and Technology Press, 2017; 136p. (in Chinese) 
[2] Yi Z Y. China Agricultural Mechanization Yearbook 2018. China Agricultural Science and Technology Press, 2018; 120p. (in Chinese)

[3] Keicher R, Seufert H. Automatic guidance for agricultural vehicles in Europe. Computers and Electronics in Agriculture, 2000; 25(1-2): 169-194.

[4] Bakker T, Wouters H, Asselt K V, Bontsema J, Tang L, Müller J, et al. A vision based row detection system for sugar beet. Computers and Electronics in Agriculture, 2008; 60(1): 87-95.

[5] Bakker T, Asselt K V, Bontsema J, Müller J, van Straten G. Autonomous navigation using a robot platform in a sugar beet field. Biosystems Engineering, 2011; 109(4): 357-368.

[6] García-Santillán I D, Montalvo M, Guerrero J M, Pajares G. Automatic detection of curved and straight crop rows from images in maize fields. Biosystems Engineering, 2017; 156: 61-79.

[7] Zhang Q, Chen M E S, Li B. A visual navigation algorithm for paddy field weeding robot based on image understanding. Computers \& Electronics in Agriculture, 2017; 143: 66-78.

[8] Burgos-Artizzu X P, Ribeiro A, Tellaeche A, Pajares G, Fernández-Quintanilla G. Analysis of natural images processing for the extraction of agricultural elements. Image and Vision Computing, 2010; 28(1): 138-149.

[9] Montalvo M, Pajares G, Guerrero J M, Romio J, Guijarro M, Ribeiro A, et al. Automatic detection of crop rows in maize fields with high weeds pressure. Expert Systems with Applications, 2012; 39(15): 11889-11897.

[10] Kaizu Y, Imou K. A dual-spectral camera system for paddy rice seedling row detection. Computers and Electronics in Agriculture, 2008; 63(1): 49-56.

[11] Tang L, Tian L F, Steward B L. Classification of broadleaf and grass weeds using Gabor wavelets and an artificial neural network. Transactions of the ASAE, 2003; 46(4): 1247-1254.

[12] Jeon H Y, Tian L F, Zhu H. Robust crop and weed segmentation under uncontrolled outdoor illumination. Sensors, 2011; 11(12): 6270-6283.

[13] Swain K C, NøRremark M, JøRgensen R N, Midtiby H S, Green O. Weed identification using an automated active shape matching (AASM) technique. Biosystems Engineering, 2011; 110(4): 450-457.

[14] Hyyti H, Kalmari J, Visala A. Real-time detection of young spruce using color and texture features on an autonomous forest machine. In: 2013 International Joint Conference on Neural Networks. Dallas: IEEE, 2013; pp.1-8.

[15] Hu L, Lin C X, Luo X W, Yang W W, Xu Y, Zhou H, et al. Design and experiment on auto leveling control system of agricultural implements. Transactions of the CSAE, 2015; 31(8): 15-20. (in Chinese)

[16] Zhao R M, Hu L, Luo X W, Zhou H, Du P, Tang L M, et al. A novel approach for describing and classifying the unevenness of the bottom layer of paddy fields. Computers and Electronics in Agriculture, 2019; 158: 183-188

[17] Søgaard H T, Olsen H J. Determination of crop rows by image analysis without segmentation. Computers and Electronics in Agriculture, 2003; 38(2): 141-158.
[18] Jiang G, Wang Z, Liu H. Automatic detection of crop rows based on multi-ROIs. Expert Systems with Applications, 2015; 42(5): 2429-2441.

[19] Zhang X Y, Li X N, Zhang B H, Zhou J, Tian G Z, Xiong Y J, et al Automated robust crop-row detection in maize fields based on position clustering algorithm and shortest path method. Computers and Electronics in Agriculture, 2018; 154: 165-175.

[20] Gao G Z, Wang Y Y, Zheng F X. The effect of light intensity on the growth and development of late rice at different closure periods - An empirical study on Chen Yongkang's suitable closure period for high-yield late rice (1). Acta Meteorologica Sinica, 1966; 2: 35-42

[21] Wang W J. Crop cultivation. Science and Technology Literature Publishing House, 1998; 347p. (in Chinese)

[22] Jiang G Q, Yang X Y, Wang Z H, Liu H M. Crop rows detection based on image characteristic point and particle swarm optimization-clustering algorithm. Trans CSAE, 2017; 33(11): 173-178. (in Chinese)

[23] Choi K H, Han S K, Han S H, Park K H, Kim K S, Kim S H Morphology-based guidance line extraction for an autonomous weeding robot in paddy fields. Computers and Electronics in Agriculture, 2015; 113: $266-274$

[24] Liao J, Wang Y, Yin J, Zhang S, Liu L, Zhu D. Detection of seedling row centerlines based on sub-regional feature points clustering. Transactions of CSAM, 2019; 50(11): 34-41. (in Chinese)

[25] Chen Z, Li W, Zhang W Q, Li Y W, Li M, Li H. Vegetable crop row extraction method based on accumulation threshold of Hough Transformation. Transactions of the CSAE, 2019; 35(22): 314-322. (in Chinese)

[26] Otsu N. A threshold selection method from gray-level histograms. Automatica, 1975; 11(285-296): 23-27.

[27] Woebbecke D M, Meyer G E, Von Bargen K, Mortensen D A. Color indices for weed identification under various soil, residue, and lighting conditions. Transactions of the ASAE, 1995; 38(1): 259-269.

[28] Bao X M, Wang Y M. Apple image segmentation based on the minimum error Bayes decision. Transactions of the CSAE, 2006; 22(5): 122-124. (in Chinese)

[29] Liu C L. Image processing with MATLAB. Beijing: Tsinghua University Press, 2017; 450p. (in Chinese)

[30] Onyango C M, Marchant, J A. Segmentation of row crop plants from weeds using colour and morphology. Computers and Electronics in Agriculture, 2003; 39(3): 141-155.

[31] Hu L, Luo X W, Zeng S, Zhang Z G, Chen X F, Lin C X. Plant recognition and localization for intra-row mechanical weeding device based on machine vision. Transactions of the CSAE, 2013; 29: 12-18. (in Chinese)

[32] Javier G, Daniel P, Romer R. A robust partial least squares regression method with applications. Journal of Chemometrics, 2009; 23(2):78-90.

[33] Huber P J. Robust statistics. Springer Berlin Heidelberg, 2011; 380p.

[34] Lawson C, Keats J B, Montgomery D C. Comparison of robust and least-squares regression in computer-generated probability plot. IEEE Transactions on Reliability, 1997; 46(1): 108-115. 